\title{
A Load Balance Clustering Routing Algorism Based on SOM
}

\author{
Shan Zhong, Guihua Wang, Xiaohui Leng, Xiaona Wang, Lian Xue, Yue Gu
}

Computer Science and Engineering College, Changshu Institute of Technology, Suzhou, China.

Email: sunshine-620@163.com

Received September $7^{\text {th }}, 2012$; revised October $9^{\text {th }}, 2012$; accepted October $20^{\text {th }}, 2012$

\begin{abstract}
In order to solve the uneven node load in the tradition clustering routing protocols, a new clustering algorism based on SOM is proposed. Firstly, the network radio model and the energy consumption model are defined. A new algorism using SOM to form the cluster and select the cluster head is defined. In the clustering node remain energy and the Euclidean distance from cluster head to the cluster member are considered. The experiment shows our method has the longer life cycle and less total energy consumption. It is an effective clustering protocol.
\end{abstract}

Keywords: SOM; Routing Protocol; Clustering; Load Balance

\section{Introduction}

WSN (Wireless Sensor Network) is composed of amounts of sensor nodes with low-power and limited-processing capability random deployed in the monitoring area [1].

The routing protocol is the main research of WSN, the clustering protocol as a hierarchical protocol is more extensive than the other types of protocols, the main idea is the network can be divided to several clusters, every cluster has two types of nodes such as the cluster head and the cluster member, the cluster head is responsible for collecting, managing and fusing the data from cluster member and finally sending to sink node.

LEACH protocol (Low-Energy Adaptive Clustering Hierarchy) [2] is introduced by Heinzelman, and it is a first clustering routing protocol, the operation of the protocol can be described as wheels, every wheel contains clustering and data transmission, but the selection of the cluster head not considering the energy factor leading the uneven node energy consumption.

LEACH-C protocol [3] using the centralized control strategy, the sink node operates the simulation annealing algorism to cluster, but having the big energy consumption.

HEED [4], TEEN [5] and PEGASIS [6] are all the improvement on the LEACH protocol, but they all exist the big energy consumption for clustering.

In order to solve the above problems, we propose a new method based on SOM algorism, the SOM is used to obtain the clusters and the cluster heads.

\section{Radio Model}

The network model is given the following condition:
1) The base station is fixed and located far from the sensor node.

2) All the sensor nodes in the network are homogeneous and energy constrained, the node is fixed and can not move.

3) The communication link is symmetry, when the transmit power is known, the receiving node can estimate the distance from between them.

4) According the distance from the receiving node, the sending node can adjust transmit power to save the energy.

For the main energy consumption of wireless sensor network is from data communication, so missing the data fusion and managing, the energy consumption model can be represented as follows:

$$
\begin{gathered}
E_{\text {trans }}(n, d) \\
= \begin{cases}n^{*}\left(E_{\text {elec }}+\varepsilon_{\text {fimp }}{ }^{*} d^{2}\right) & d<d_{0} \\
n^{*}\left(E_{\text {elec }}+\varepsilon_{\text {amp }}{ }^{*} d^{4}\right) & d \geq d_{0}\end{cases} \\
E_{\text {rec }}=n^{*} E_{\text {elec }}
\end{gathered}
$$

In Equation (1) $E_{\text {trans }}(l, d)$ denotes the data transmit energy consumption, $E_{\text {rec }}$ denotes the data receiving energy consumption, $n$ is the bit of data packet. $E_{\text {elec }}$ is the wireless transceiver circuit energy consumption, $\varepsilon_{\text {fimp }}$ and $\varepsilon_{\text {amp }}$ is the amplifier circuit power coefficient for free-space model and multi-path fading model, respectively. $d$ is the distance between the sending node and the receiving node. 


\section{The Clustering Algorism}

\subsection{SOM}

SOM (Self-Organizing Feature Map) [7] is proposed by Kohonen, it is main goal is to realize the input signal model of any dimension changing to one dimension or two dimension, and to adapt the change in a topological and orderly manner.

The SOM contains input layer and output layer as Figure 1.

\subsection{SOM Clustering Algorism}

Initialize: The number of input neural, the number of output neurons, weight vector

$W_{i j}(0)(1 \leq i \leq n, 1 \leq j \leq m)$, iteration time $t=1$, the max iteration time $T$;

Step 1: The current iteration time $t=t+1$, input the sample $s_{i}=\left\{x, y, e_{\text {remain }}\right\}$ and the weight value $W_{i j}(0)$. All the samples and the weight value $W_{i j}(0)$ are normalized.

Step 2: Find the successful neuron. Find the successful output neuron according to the Equation (3) with the smallest Euclidean distance.

$$
\operatorname{dist}_{i j}(t)=\min \sum_{i}^{n}\left(w_{i j}(t)-x_{i}(t)\right)^{2} \quad j=1,2, \cdots, m
$$

In Equation (3), the output neuron which has the smallest $\operatorname{dist}_{i j}(t)$ is the successful neuron.

Step 3: Renew the weight. Renew the weight of successful neuron and its neighbor area according to the Equation (4):

$$
w_{i j}(t+1)= \begin{cases}w_{i j}(t)+h(t) \hbar_{i j}(t)\left(x_{i}-w_{i j}(t)\right) & i \in N_{j}(t) \\ w_{i j}(t) & i \notin N_{j}(t)\end{cases}
$$

In Equation (4), $h(t)$ is the studying rate, $\hbar_{i j}(t)$ is

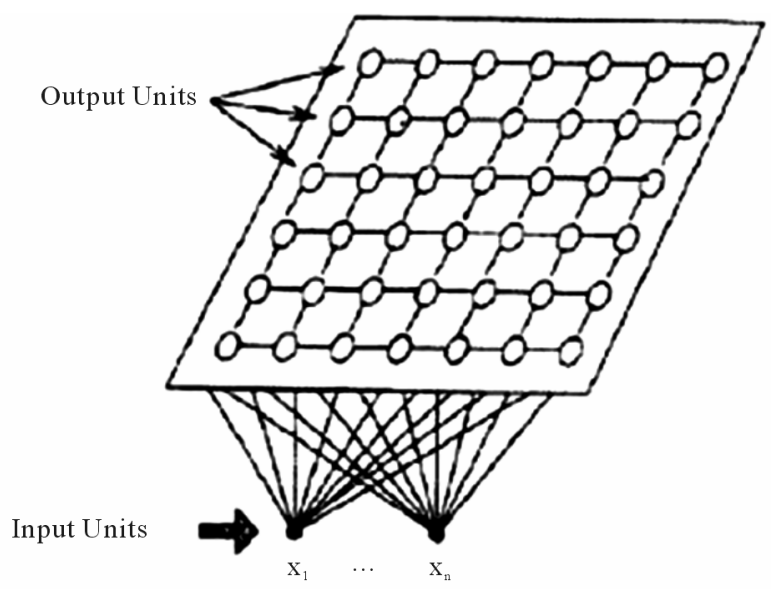

Figure 1. The structure of SOM. the neighbor area function.

Step 4: Renew the studying rate and the neighbor area function according to the following equations.

$$
\begin{aligned}
h(t) & =h_{0} \exp (-t / t 1) \\
\hbar_{i j}(t) & =\exp \left(-d^{2} / 2 \sigma^{2}\right)
\end{aligned}
$$

In the above equations, $h(t)$ and $\hbar_{i j}(t)$ is dynamically descending, and according the experience $h_{0}$ is assigned $0.1, t 1$ is assigned 1000 , so $h(t)$ is between 0.01 and $0.1, \hbar_{i j}(t)$ is a Gaussian function and the variable $\sigma$ is a function descending with the iteration time. $\sigma$ is represented by the Equation (7):

$$
\sigma(t)=\sigma_{0} \exp (t / t 1)
$$

In the Equation (7), the value of $t 1$ is also 1000 .

Step 5: If the studying rate is descent to 0 or the current iteration time is the max value $T$, the cluster numbers and its responding center $c=\left\{c_{1}, c_{2}, \cdots, c_{k}\right\}$ can be obtained, else go to Step 2.

Step 6: Obtain the cluster head. Assign all the cluster center to the really sensor node according Equation (8):

$$
\left\|x_{i}-c_{h}\right\|<\forall j\left\|x_{j}-c_{h}\right\|(1 \leq i, j \leq n)
$$

In Equation (8), $c_{h}(1 \leq h \leq k)$ is one of the cluster centers from the set $c$, so the cluster center is assigned to $x_{i}$, and then $x_{i}$ is the cluster head.

Step 7: Form the clusters. Assign every sensor node to the cluster according to the smallest Euclidean distance from the cluster head.

When the cluster is formed and the cluster head is selected, the cluster is not changed any more, but there existed an energy threshold, if the energy of the cluster head is lower than the threshold, it will broadcast a message to tell all the cluster members, and the cluster head will be reselect to according the algorism.

\section{Simulation Experiment}

Using Matlab as the simulation platform, and the experiment parameters are showed as in Table 1:

Figure 2 shows numbers of remain live nodes according to the wheels:

From Figure 2 we can find our method has the longer network life cycle than LEACH and LEACH-C, and the first death node in our method is appeared in 350 wheels, where LEACH and LEACH-C are 150 and 170, respectively. It proves that our method has balanced the load of cluster node by making the near distance from the cluster head.

Figure 3 shows the total energy consumption for the three protocols, which shows our method has the smallest energy consumption, and this is because the formed cluster will not be changed, so avoiding the periodical forming cluster of other protocols. 
Table 1. Experiment parameters.

\begin{tabular}{cc}
\hline Paramter & Value \\
\hline Sensor area & $(0,0)-(100,100) \mathrm{m}$ \\
Node initial energy & $4 \mathrm{~J}$ \\
Node number & 100 \\
Station position & $(25,250) \mathrm{m}$ \\
$\varepsilon_{f s}$ & $30 \mathrm{pJ} / \mathrm{bit}^{*} \mathrm{~m}^{-2}$ \\
$\varepsilon_{m p}$ & $0.4 \mathrm{pJ} / \mathrm{bit}^{*} \mathrm{~m}^{-4}$ \\
$\varepsilon_{m p}$ & $30 \mathrm{~nJ} / \mathrm{bit}$ \\
$E_{d a}$ & $10 \mathrm{~nJ} / \mathrm{bit}$ \\
Data packet & $600 \mathrm{~B}$ \\
\hline
\end{tabular}

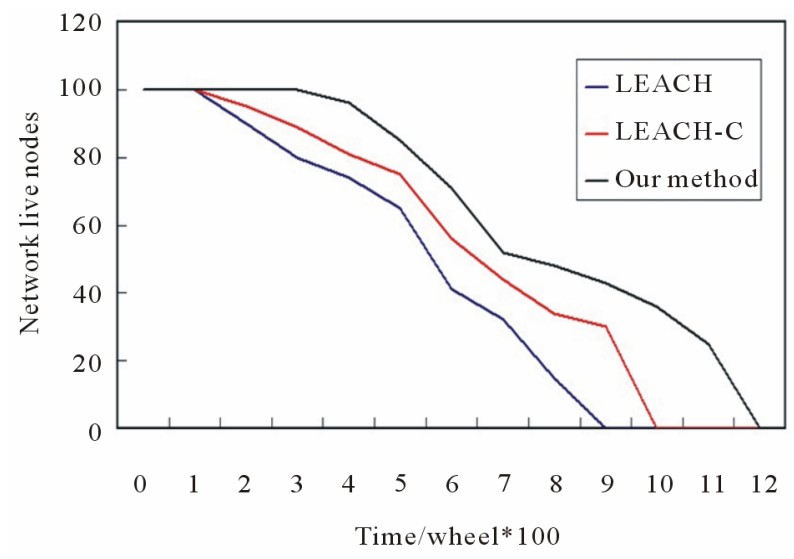

Figure 2. Remain live nodes.

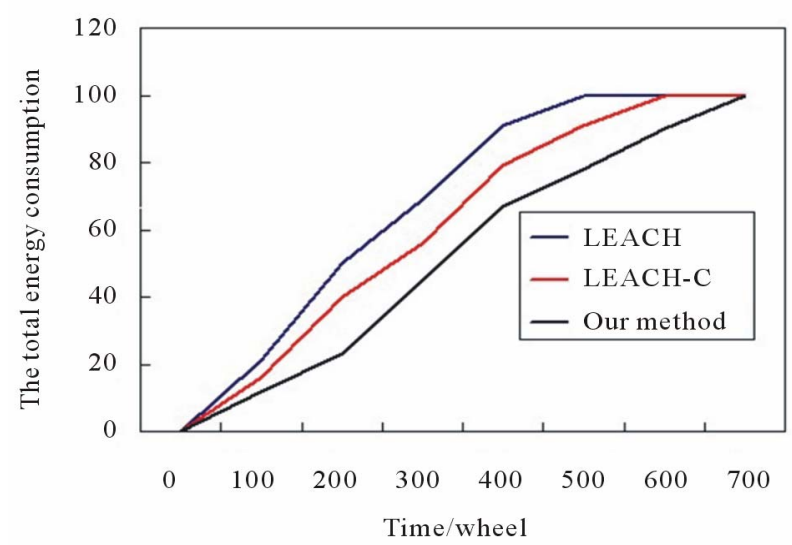

Figure 3. The total energy consumption.

\section{Conclusion}

In the WSN, the node is usually powered battery and can not be supplemented, so the effective routing method is needed to delay the network life cycle. Therefore, we proposed a cluster algorism based on SOM is introduced. The algorism is designed based on two types' information such as node position and remain energy. And after the operation of the algorism we can get the cluster and the cluster head. The experiment shows our method has the longer life cycle and less total energy consumption. It is an effective clustering protocol.

\section{REFERENCES}

[1] C. Y. Chong and S. P. Kumar, "Sensor Networks: Evolution, Opportunities, and Challenges," Proceedings of the IEEE, Vol. 91, No. 8, 2003, pp. 1247-1256. doi:10.1109/JPROC.2003.814918

[2] W. Heinzelman, A. Chandrakasan and H. Balak Rishnan, "Energy-Efficient Communication Protocol for Wireless Micro Sensor Networks," Proceedings of the 33rd Hawaii International Conference on System Science, Hawaii, 4-7 January 2000, pp. 1247-1256.

[3] W. Heinzelman, "An Application-Specific Protocol Architecture for Wireless Microsensor Networks," IEEE Transactions on Wireless Communications, Vol. 1, No. 4, 2002, pp. 660-670. doi:10.1109/TWC.2002.804190

[4] O. Youni and S. Fahmy, "HEED: A Hybrid, EnergyEfficient, Distributed Clustering Approach for Ad Hoc Sensor Networks," IEEE Transactions on Mobile Computing, Vol. 3, No. 4, 2004, pp. 366-379. doi:10.1109/TMC.2004.41

[5] A. Manjeshwar and D. P. Agrawal, "TEEN: A Routing Protocol for Enhanced Efficiency in Wireless Sensor Networks," Proceedings of 15th IEEE International Parallel and Distributed Processing Symposium, San Francisco, 23-27 April 2001, pp. 2009-2015.

[6] S. Lindsey and C. S. Raghavendra, "PEGASIS: Power Efficient Gathering in Sensor Information Systems," Proceedings of the IEEE Aerospace.

[7] T. Kohonen, "Self-Organizing Maps," 2nd Edtion, Springer-Verlag, Berlin, 1997. 